\title{
ÉTUDE EXPÉRIMENTALE DE L'EFFET FARADAY INVERSE DANS LES PLASMAS
}

\author{
J. DESCHAMPS, M. FITAIRE et M. LAGOUTTE \\ Laboratoire de Physique des Plasmas $(*)$ \\ Université de Paris-Sud, Centre d'Orsay, 91405-Orsay
}

(Reçu le 16 décembre 1971, révisé le 22 mars 1972)

\begin{abstract}
Résumé. - Les électrons d'un plasma soumis à l'action du champ électrique d'une onde polarisée circulairement possèdent chacun un moment magnétique et créent ainsi une aimantation globale. La mise en évidence expérimentale de cet effet, réciproque de l'Effet Faraday classique, conduit en particulier à montrer que l'induction magnétique produite est proportionnelle au carré du champ électrique qui lui donne naissance. Des impulsions de quelques mégawatts d'ondes hyperfréquence à $3000 \mathrm{MHz}$ ont été utilisées et les variations du flux magnétique créé ont été détectées à l'aide d'une bobine de fils conducteurs disposée autour du plasma.
\end{abstract}

\begin{abstract}
When subjected to the electric field of a circularly polarized wave, the plasma electrons acquire a magnetic moment and thus create a global magnetization. An experiment demonstrating this effect shows that the magnetic induction thus produced is proportional to the square of the applied electric field. Pulsed high frequency signals of several megawatts at $3.000 \mathrm{MHz}$ were applied to the plasma and the variation of the magnetic flux was detected with a coil placed around the plasma.
\end{abstract}

I. Introduction. - Un électron qui décrit une orbite circulaire possède un moment magnétique perpendiculaire à son plan de rotation et une assemblée de tels électrons crée de ce fait une aimantation induite globale. Ce mouvement de giration peut en particulier être obtenu en faisant agir sur les électrons un champ électrique à polarisation circulaire. On réalise ainsi une situation que les physiciens du solide ont montré être réciproque de l'Effet Faraday classique [1]-[2], qu'ils ont dénommé Effet Faraday Inverse (EFI) et mis en évidence [3].

Dans le cas des plasmas cet EFI a été prévu et il a été montré qu'il s'agit bien d'un effet réciproque de l'Effet Faraday direct [4]; ce qui suit est relatif à son étude expérimentale, étude dont une partie a déjà été résumée par ailleurs [5].

A un électron tournant sur un cercle de rayon $r_{0}$ sous l'action d'un champ à polarisation circulaire d'amplitude $E_{0}$, est associé un moment magnétique $\mathbf{m}_{0}$ dont le module est égal à $\left(e E_{0} / m \omega^{2}\right)^{2} e \omega / 2$, où $e$ et $m$ sont respectivement la charge et la masse de l'électron et $\omega$ la pulsation de l'onde. Si la densité volumique des électrons est $n$, le moment magnétique par unité de volume est alors $\mathbf{M}=n \mathbf{m}_{0}$. Une induction magnétique permanente $B$ est ainsi créée ; pour un volume unité elle est donnée par : $B=\left(e / 2 m c^{2}\right)\left(\omega_{p}^{2} / \omega^{3}\right) E_{0}^{2}$ où $\omega_{p}^{2}=n e^{2} / m \varepsilon_{0}, \varepsilon_{0}$ étant la permittivité du vide. Dans le cas des électrons d'un plasma de volume $v$, la

(*) Associé au CNRS. densité électronique n'est pas uniforme et il y a donc lieu d'en tenir compte en remplaçant $\omega_{p}^{2}$ par $\vartheta<\omega_{p}^{2}>$, où $<\omega_{p}^{2}>$ est la valeur moyenne de $\omega_{p}^{2}$ dans $\vartheta$. Nous appellerons $\mathfrak{B}$ l'induction totale ainsi créée qui est donc, pour un plasma donné, de la forme $\mathcal{B}=\alpha E_{0}^{2}$. On notera que le champ $E_{0}$ est le champ auquel les électrons sont soumis : à cause des effets de polarisation du plasma, $E_{0}$ est différent du champ $E_{\mathrm{i}}$ appliqué et seul le calcul de la constante diélectrique de ce plasma permettrait de connaître le rapport $E_{0} / E_{\mathrm{i}}$. Notre étude étant limitée à la mise en évidence de l'EFI, un tel calcul n'est pas, ainsi que nous le verrons, nécessaire.

On calcule ainsi que pour $E_{0}=10^{6} \mathrm{~V} / \mathrm{m}$ et une densité électronique de $10^{12} \mathrm{~cm}^{-3}$, l'aimantation créée est de l'ordre de $5 \times 10^{-5} \mathrm{G} / \mathrm{cm}^{3}$ pour une onde de $3000 \mathrm{MHz}$ (dans les mêmes conditions $r_{0} \simeq 0.5 \mathrm{~mm}$ ). De tels niveaux de champ ne peuvent être obtenus en régime continu et l'induction $\mathfrak{B}$ est donc modulée à la fréquence de répétition de l'onde utilisée. Il en résulte une situation favorable dont on tire parti en mesurant non pas $\mathcal{B}$ mais les variations de son flux à travers une bobine dans laquelle est produit le plasma. Avantageuse quant à la sensibilité de détection, cette méthode rend difficile les mesures absolues de $\Re$ : il est impossible d'éviter la présence de corps conducteurs au voisinage du plasma, corps au travers desquels doit diffuser le champ. Bien que le calcul de $\mathfrak{B}$ soit théoriquement possible dans cette situation [6], la forme nécessairement compliquée 
des conducteurs conduit en fait à des difficultés de calcul à peu près insurmontables.

On remarquera par ailleurs que la relation (1) ne donne en toute rigueur que la valeur stationnaire de B puisqu'elle néglige le courant de déplacement $\partial \mathbf{D} / \partial t$ dans $\operatorname{rot} \mathbf{B}=\mu_{0}(\operatorname{rot} \mathbf{M}+\partial \mathbf{D} / \partial t)$. Dans la configuration expérimentale choisie (voir en II) elle reste néanmoins applicable même pendant la phase de croissance ou de décroissance de $\mathbf{M}$ : les termes associés aux variations de $\mathbf{D}$ fluctuent à la fréquence de l'onde incidente et ont donc une profondeur de pénétration dans les conducteurs entourant le plasma inférieure à l'épaisseur de ceux-ci ; en outre le dispositif de détection utilisé ne peut lui-même répondre à ces fréquences.

Un certain nombre d'électrons atteignent à chaque instant la paroi du récipient dans lequel est produit le plasma ; ce sont, si on néglige les collisions contre les neutres, ceux dont le centre de rotation est à une distance de la paroi inférieure au rayon du cercle décrit. Si ces électrons ne sont pas absorbés par la paroi, ils parcourent, avant d'y retomber un arc de cercle dont la longueur dépend de la direction dans laquelle ils ont été précédemment réémis par cette paroi. Il est facile de se convaincre de ce que ces électrons tendent à créer une induction magnétique de sens contraire à celle créée par les électrons dans le volume du plasma. Le rapport de ces deux inductions est au plus égal au rapport du rayon de giration des électrons à celui du récipient qui contient le plasma, c'est-à-dire, en ce qui nous concerne (voir I et II), inférieur à $2 \times 10^{-2}$.

II. Montage expérimental. - La mise en évidence de l'EFI suppose l'utilisation de signaux électromagnétiques à polarisation circulaire ayant un champ électrique associé aussi grand que possible et une fréquence pas trop élevée (terme en $\omega^{-3}$ ). Un bon compromis entre différentes approches du problème consiste à choisir de travailler dans le domaine des hyperfréquences (HF), domaine dans lequel existent des générateurs de grande puissance dont les signaux peuvent être aisément couplés à un plasma. Dans le montage expérimental utilisé l'onde électromagnétique sert à la fois à produire le plasma et à y exciter l'EFI dont la mise en évidence est réalisée par la mesure des variations de flux dans une bobine disposée autour de ce plasma.

Le générateur hyperfréquence utilisé est un klystron amplificateur (Thomson-CSF, F. 2042) qui fournit pendant $12 \mu$ s une puissance maximale instantanée de $23 \mathrm{MW}$ à $3000 \mathrm{MHz}$, à une fréquence de répétition de l'ordre de $7 \mathrm{~Hz}$. Ce klystron transmet sa puissance à travers deux guides rectangulaires fusionnés en aval en un seul guide par une jonction en $Y$. L'un des deux guides est déformable dans la direction de son grand côté de façon à permettre l'accord de phase des deux ondes qui se composent à la sortie de. la jonction.
De façon à minimiser le taux de réflexion de la ligne de propagation sur laquelle débite le klystron, on interpose entre celle-ci et le plasma un coupleur directif dont le couplage est de $3 \mathrm{~dB}$. Cette configuration permet en outre de mesurer commodément la puissance $\mathrm{HF}$ incidente et la puissance $\mathrm{HF}$ réfléchie par le plasma. Dans les expériences qui seront décrites par la suite, un niveau de puissance crête de l'ordre de quelques mégawatts a été utilisé, niveau pour lequel le découplage ainsi réalisé s'est révélé suffisant compte tenu du montage de la ligne de propagation qui lui fait suite et qui comprend successivement (Fig. 1) :

- une transition guide rectangulaire-guide cylindrique $t_{1}$ permettant de passer du mode fondamental de l'un $\left(\mathrm{TE}_{01}\right)$ au mode fondamental de l'autre $\left(\mathrm{TE}_{11}\right)$;

- un Té de correction sur guide cylindrique ;

- un polariseur circulaire $\mathrm{P}_{1}$;

- une cellule à plasma $\mathrm{Z}$;

- un polariseur $\mathrm{P}_{2}$ identique à $\mathrm{P}_{1}$ et croisé avec celui-ci ;

- une transition $t_{2}$ guide circulaire-guide rectangulaire ;

- un coupleur directif $\mathrm{C}_{2}$ permettant de détecter une partie du signal transmis;

- une charge adaptée à eau $\mathrm{Z}_{\mathbf{c}_{1}}$.

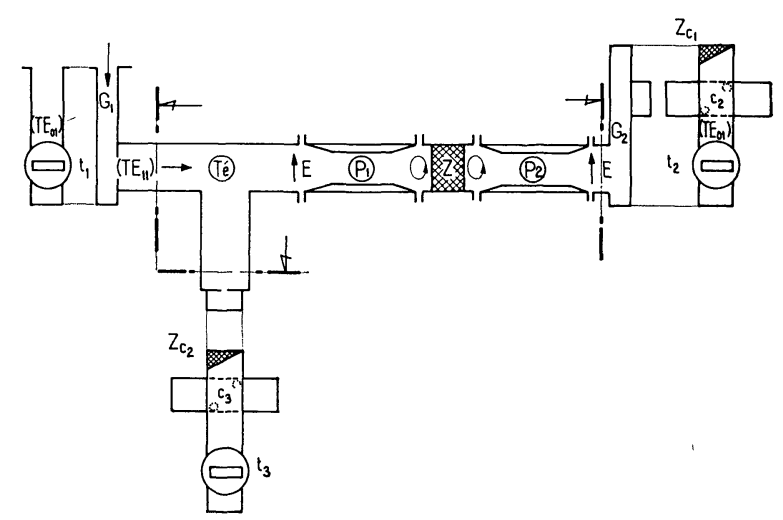

Fig. 1. - Schéma de la ligne de propagation utilisée en aval du coupleur directif à $3 \mathrm{~dB} ; \mathrm{G}_{1}$ et $\mathrm{G}_{2}$ : guide rectangulaire (CNET, $\left.\lambda_{7}\right): P_{1}$ et $P_{2}:$ polariseurs $; t_{1}, t_{2}$ et $t_{3}:$ transitions guide rectangulaire-guide cylindrique; $C_{2}$ et $C_{3}$ : coupleurs directifs; $Z_{c_{1}}$ et $\mathrm{Z}_{\mathrm{c}_{2}}$ : charges adaptées à eau; $\mathrm{Z}$ : cellule à plasma (Fig. 2).

Le principe de fonctionnement et la réalisation du Té et du polariseur ont été décrits par ailleurs [7]. Rappelons que le rôle du Té est d'éviter les effets de dépolarisation de l'onde dus à l'utilisation de charges désadaptées et que, dans le cas où $Z$ est complètement réfléchissante, le TOS en amont du Té est compris entre 1,04 et 1,52 suivant la position de cette cellule avec alors une ellipticité de l'onde comprise entre 1,09 et 1,39 .

Le polariseur $\mathrm{P}_{2}$ est croisé avec $\mathrm{P}_{1}$ de façon à : 1) reformer une onde à polarisation rectiligne, 2) utiliser un coupleur directif et une charge à eau de réalisations classiques. 
La cellule à plasma (Fig. 2) est un cylindre en verre pyrex fermé à ses deux extrémités, cylindre qui est inséré dans un tronçon de guide circulaire. Deux

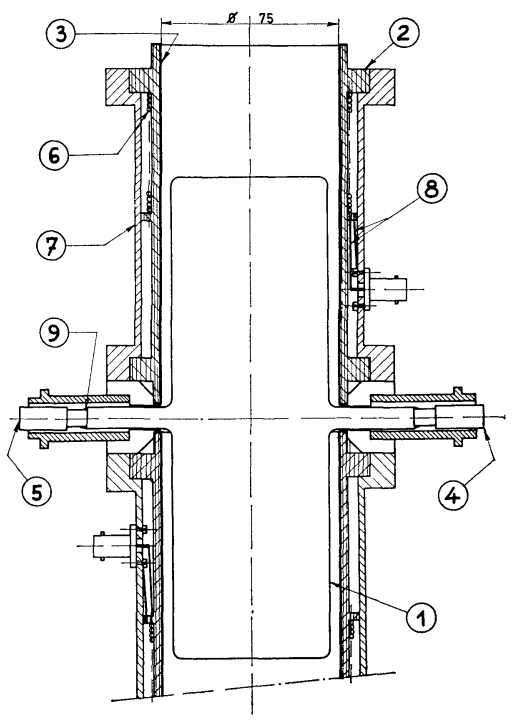

FIG. 2. - Cellule à plasma ; $1:$ tube en verre pyrex $(\varnothing$ ext $=65$, $\left.\varnothing_{\text {int }}=60, \mathrm{~L}=200\right) ; 2:$ guide en nylon $\left(\varnothing_{\text {int }}=75\right) ; 3$ : dépôt de cuivre (épaisseur 20 à $30 \mu) ; 4$ : voie de pompage et d'admission de gaz ; 5 : fenêtre de visée optique ; 6 : cent spires jointives, fil de cuivre émaillé ; $7:$ demi-cylindre de cuivre feuilleté dans le sens de la longueur; 8 : connexions de la bobine ; 9 : raccord verre-métal.

orifices cylindriques diamétralement opposés permettent, l'un l'observation optique du plasma, l'autre le pompage et le remplissage de cette cellule à l'aide d'hélium; de pureté commerciale courante, ce gaz a été utilisé tout au long de l'étude. Le banc de pompage comprend une pompe préliminaire à palettes et une pompe à diffusion d'huile.

Le tronçon de guide circulaire dans lequel est insérée la cellule à plasma est constitué de deux couronnes en nylon, chacune métallisée intérieurement à l'aide d'un dépôt de cuivre de 10 à $20 \mu$ d'épaisseur. Ce dépôt a pour but d'assurer la continuité des champs de l'onde électromagnétique dans la ligne de propagation, onde dont la profondeur de pénétration est de l'ordre de $1,2 \mu$. Autour de chacune de ces couronnes est disposée une bobine formée par cent spires de fil émaillé $(5 / 10 \mathrm{~mm})$ aux bornes de l'une desquelles on mesure les variations du flux magnétique qui la traverse.' L'épaisseur de peau relative à ces variations est de l'ordre de $100 \mu$ pour un temps de montée du signal de l'ordre de la microseconde ; cette épaisseur est donc nettement plus grande que celle du dépôt de cuivre. Une telle bobine est prolongée par un câble coaxial et fermée sur une résistance $(\simeq 500 \Omega)$ qui permet d'obtenir l'amortissement critique de l'ensemble avec alors un temps de réponse de l'ordre de la microseconde.

De façon à prévenir des claquages intempestifs dans la ligne de propagation, celle-ci est après pom- page remplie d'hexafluorure de soufre $\left(\mathrm{SF}_{6}\right)$ sous une pression de 2 bars.

III. Résultats expérimentaux. - A) MISE EN ÉVIDENCE DU PHÉNOMÈNE. - Le polariseur fournissant une onde à polarisation circulaire, on enregistre le signal $\mathrm{S}$ recueilli aux bornes de la bobine ainsi que le signal HF incident pendant une impulsion (Fig. 3).

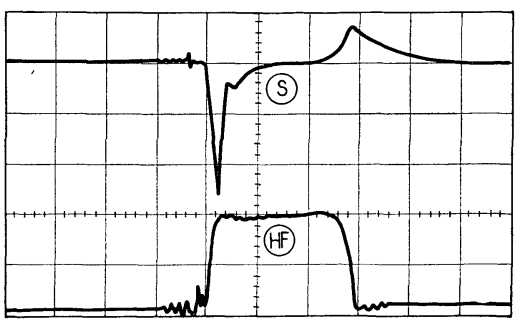

Fig. 3. - Oscillogrammes représentant le signal $\mathrm{S}$ détecté aux bornes de la bobine et l'impulsion d'onde hyperfréquence (HF) utilisée ; bal. horiz. : $4 \mu \mathrm{s} /$ carreau ; bal. vert. : $1 \mathrm{~V} /$ div ; puissance HF : $1,5 \mathrm{MW}$.

On constate que $\mathrm{S}$ présente deux pics de signes opposés, le premier de ceux-ci correspondant au début de l'impulsion et le deuxième à la fin de cette même impulsion.

Pour s'assurer que le signal $\mathrm{S}$ est dû à l'action à travers la bobine du champ magnétique variable dans le temps créé par EFI, on réalise alors successivement les quatre expériences suivantes à partir d'une situation expérimentale initiale donnée que nous appellerons $e_{0}$ :

1. On inverse le sens du bobinage.

2. On revient à $e_{0}$ puis on tourne le polariseur de $\pi / 2$ (ce qui a pour effet de transformer une onde circulaire droite en onde circulaire gauche).

Dans ces deux cas on observe que $\mathrm{S}$ change de signe en passant de $e_{0}$ à la situation considérée.

3. A pression très basse ( $\lesssim 10^{-5}$ torr) la même onde ne produit aucun plasma et le signal $\mathrm{S}$ est alors nul ; mêmes observations pour $p \gtrsim 20$ torrs.

4. En l'absence de polariseur un plasma est produit mais aucun signal n'est détectable aux bornes de la bobine.

Les expériences 1 et 2 s'expliquent bien par l'action d'une induction magnétique variable dans le temps à travers la bobine. Dans le cas 1 , le signal $\mathbf{S}$ proportionnel à la variation de flux à travers la bobine change de signe puisque le vecteur normal à la surface de chaque spire change lui-même de sens. Dans le cas 2, les électrons tournent en sens contraire, ce qui donne naissance à une induction magnétique opposée à la précédente. Les expériences 3 et 4 montrent que seule l'action sur le plasma de l'onde polarisée circulairement crée le champ magnétique observé.

On remarque en outre sur la figure 3 , que les deux pics de S sont de hauteurs inégales. Pour comprendre ceci dérivons par rapport au temps la formule théo- 
rique donnant l'induction $B$ par unité de volume créé par EFI :

$$
\frac{\mathrm{d} B}{\mathrm{~d} t}=A \frac{\mathrm{d} E_{0}}{\mathrm{~d} t}+A^{\prime} \frac{\mathrm{d} \omega_{p}}{\mathrm{~d} t}
$$

$A$ et $A^{\prime}$ dépendant de $E_{0}$ et $\omega_{p}$. Les extrema de $\mathrm{S}$ sont donc proportionnels, toutes choses égales par ailleurs, aux variations temporelles, au début et à la fin de l'impulsion, du champ électrique polarisé circulairement et de la fréquence plasma. Les temps de montée et de descente du signal HF sont équivalents et de l'ordre de $0,5 \mu$ s ; par contre au début de l'impulsion la densité électronique a un temps caractéristique de croissance de l'ordre de la microseconde alors qu'à la fin de l'impulsion le temps caractéristique de décroissance est de l'ordre de la milliseconde (pour une pression de $10^{-2}$ torr). Ceci permet de comprendre à la fois l'origine des signes opposés des deux pics de $\mathrm{S}$ et la plus grande amplitude du premier.

B) ETUDE COMPLÉMENTAIRE EN FONCTION DE LA PRESSION DU GAZ NEUTRE DE LA DÉCHARGE. - On observe que le signal $\mathrm{S}$ aux bornes de la bobine est nul pour une pression inférieure à $10^{-2}$ torr environ ; il est facile de vérifier à l'aide d'un photomultiplicateur dirigé vers la cellule à plasma quu'aucune décharge n'est créée dans ces conditions, ce que confirme la mesure de la puissance $H F$ transmise $(T)$ ou réfléchie (R) par la cellule.

Le signal $\mathrm{S}$ est également nul pour une pression supérieure à $3 \times 10^{-1}$ torr et ceci bien que le plasma soit alors créé. Ce point peut être aisément éclairci si on remarque que pour de telles pressions la fréquence de collision électrons neutres est comparable à celle de l'onde électromagnétique incidente.

Les oscillogrammes de la figure 4 reproduisent les
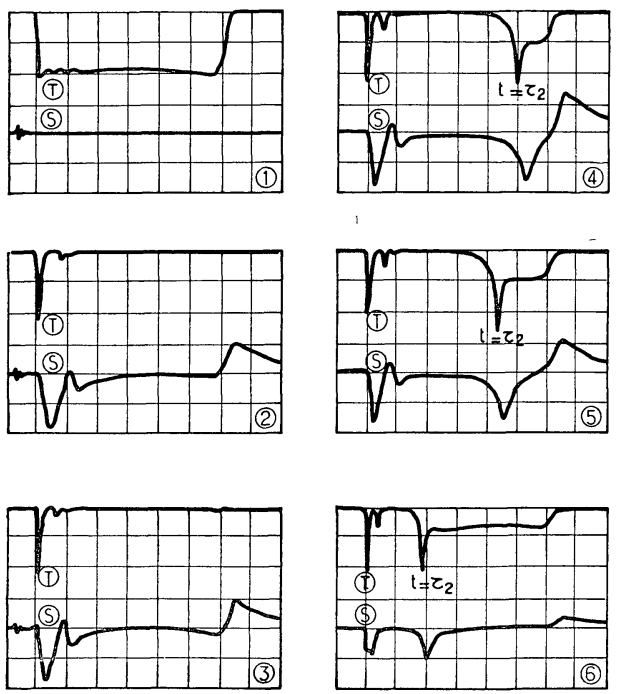

Fig. 4. - Oscillogrammes représentant le signal $\mathrm{T}$ transmis par le plasma (détecté sur le coupleur $C_{2}$ ) et le signal $S$ détecté aux bornes de la bobine pour différentes pressions d'hélium dans la cellule ; $1: 10^{-5}, 2: 5 \times 10^{-2}, 3: 6 \times 10^{-2}, 4: 8 \times 10^{-2}$, $5: 9 \times 10^{-2}$ et $6: 2 \times 10^{-1}$ torr; puissance $\mathrm{HF}: 1,5 \mathrm{MW}$; bal. horiz.; $2 \mu \mathrm{s} / \mathrm{cm}$. variations de $\mathrm{S}$ et du signal $\mathrm{T}$ transmis. Pour des pressions supérieures à $10^{-2}$ torr, $\mathrm{T}$ garde, pendant un temps $\tau_{1}$ (mesuré à partir du début de l'impulsion), la même valeur que lorsqu'il n'y a pas de plasma. C'est le temps au bout duquel le nombre d'électrons libres est tel que la fréquence de coupure du guide chargé par le plasma soit comparable à la fréquence de l'onde incidente. Ce temps $\tau_{1}$ diminue quand la pression augmente puisqu'alors la probabilité d'ionisation croît.

Pour une pression de l'ordre de $7 \times 10^{-2}$ torr, il apparaît au temps $\tau_{2}$ dans le signal $S$ de la bobine un troisième pic de même sens que le premier. Il correspond à celui-ci un autre pic dans le signal transmis, suivi d'un palier non nul jusqu'à la fin de l'impulsion. La brusque variation de $\mathrm{T}$ montre que le plasma est alors moins réfléchissant: il se développe donc dans celui-ci un champ HF plus grand qu'avant $\tau_{2}$, champ qui garde ensuite une valeur à peu près constante jusqu'à la fin de l'impulsion (l'énergie transmise et l'énergie réfléchie restent sensiblement constantes). Si l'on admet, comme nous le montrerons plus loin, que $\mathfrak{B}$ est proportionnel à $E_{0}^{2}$, $\mathrm{d} \Re / \mathrm{d} t$ est alors positif et crée donc un nouveau pic dans $\mathrm{S}$ avec le même signe que le premier.

La figure 5 montre les variations temporelles des
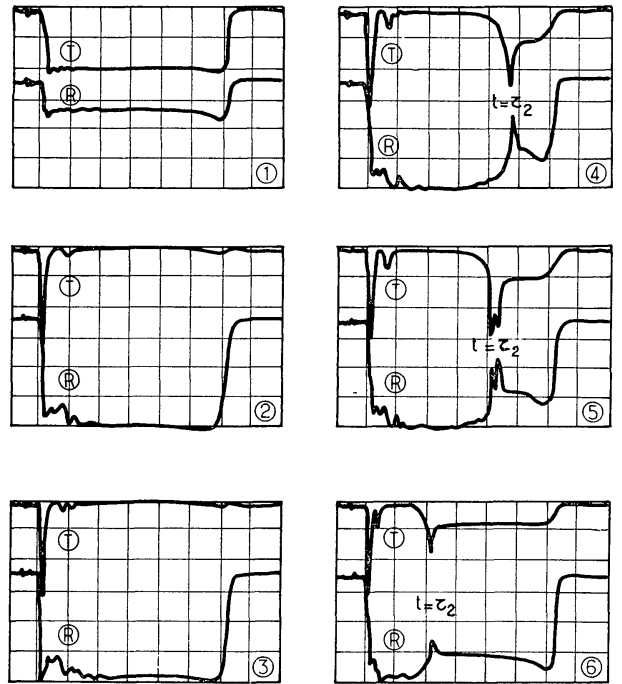

Fig. 5. - Oscillogrammes représentant le signal $\mathrm{T}$ transmis par le plasma (détecté sur le coupleur $C_{2}$ ) et le signal réfléchi $R$ (détecté sur le coupleur $\mathrm{C}_{3}$ ) pour différentes pressions d'hélium dans la cellule $: 1,2, \ldots, 6$ pressions comparab!es à celles de la figure 4 ; puissance $\mathrm{HF} \simeq 1,5 \mathrm{MW}$; bal. horiz. : $2 \mu \mathrm{s} / \mathrm{cm}$.

signaux réfléchis $\mathrm{R}$ et transmis $\mathrm{T}$ pour des pressions comparables à celles de la figure 4 . On remarque que la somme $T+R$ est à peu près constante et qu'en particulier à l'instant $\tau_{2}$ le plasma réfléchit moins et transmet plus d'énergie qu'aux instants antérieurs : il est donc bien alors soumis à un champ électrique plus élevé dont les variations sont à l'origine du troisième pic de $\mathrm{S}$. 
On remarquera en outre sur la figure 5 et plus particulièrement sur les oscillogrammes 3,4 et 5 ,' qu'il existe juste après le premier pic de $\mathrm{S}$ une remontée de ce signal ; elle correspond, à la constante de temps près de la bobine, à un ressaut dans la courbe de transmission du signal $T$. Ceci peut être interprété comme précédemment.

C) Confirmation DE L'EXISTENCE DE L'EFI : ÉTUde EN FONCTION DU TAUX DE POLARISATION DE L'ONDE. - La proportionnalité de $\mathfrak{B}$ à $E_{0}^{2}$ ne peut être mise en évidence par l'étude des variations de $S$ à puissance incidente variable. Il est en effet très difficile de modifier le champ électrique au sein du plasma d'une manière contrôlable, la densité électronique, et donc les effets de polarisation du plasma, dépendant d'une façon non connue de la puissance électromagnétique incidente.

L'étude a été réalisée de la manière suivante : à pression et à puissance HF constantes, on modifie le taux de polarisation de l'onde en faisant tourner le polariseur autour de son axe ; pour chaque position du polariseur l'amplitude de $\mathrm{S}$ est mesurée et reportée en fonction de l'ellipticité de l'onde dont la valeur peut être aisément calculée. Soit en effet $E_{i} \sqrt{2} \cos \omega t$ l'amplitude du champ électrique de l'onde polarisée rectilignement à l'entrée du polariseur et $\theta$ l'angle de ce champ avec la bissectrice OX des axes principaux $\mathrm{O} x$ et $\mathrm{O} y$ du polariseur (Fig. 6).

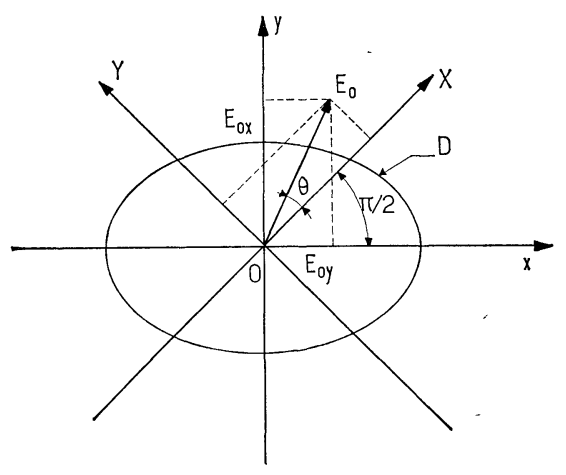

FIG. 6. - Section droite $\mathrm{D}$ du polariseur d'axes principaux $\mathrm{O} x$ et $\mathrm{O} y$ et décomposition du champ électrique $E_{0}$ de l'onde.

A la sortie de celui-ci, la composante selon $\mathrm{O} y$ est en retard de $\lambda_{g} / 4$ par rapport à celle selon $\mathrm{O} x$ et l'on a, en supposant un champ électrique incident uniforme :

$$
\left\{\begin{array}{l}
E_{x}=E_{i}(\cos \theta-\sin \theta) \cos \omega t \\
E_{y}=E_{i}(\cos \theta+\sin \theta) \cos \left(\omega t-\frac{\pi}{2}\right)
\end{array}\right.
$$

L'onde émergente a une polarisation qui dépend de la valeur de $\theta$ : elle est circulaire gauche pour $\theta=k \pi$, droite pour $\theta=\pi / 2+k \pi$, rectiligne pour $\theta=\pi / 4+k \pi$ où $k$ est un entier. Pour toute autre valeur de $\theta$ la polarisation est elliptique droite ou gauche, les axes de l'ellipse correspondant aux axes principaux du polariseur.

Ceci étant, on montre en annexe que l'induction $\mathcal{B}$ créée dans le plasma par une telle onde dont le champ électrique y a l'amplitude $E_{0}$ est de la forme :

$$
\mathcal{B}=\beta E_{0}^{2} \cos 2 \theta \mathbf{u}
$$

où $\beta$ est une constante pour un plasma donné et où $u$ est le vecteur unitaire porté par l'axe du guide dans la direction de propagation de l'onde. Pour une polarisation rectiligne l'induction créée est bien nulle; elle est maximum pour une polarisation circulaire.

Compte tenu de la manière dont la relation (3) est établie, il suffit donc, pour montrer que le champ magnétique mesuré est proportionnel au carré du champ électrique de l'onde polarisée circulairement, de mettre en évidence la proportionnalité de $\mathbf{B}$ à $\cos 2 \theta$.

Rappelons que l'expérience donne accès à $\partial \mathscr{B} / \partial t$ et non à $\mathfrak{B}$; on mesure donc, compte tenu de (3)

$$
S=\left[A E_{0} \frac{\partial E_{0}}{\partial t}+B E_{0}^{2} \frac{\partial \omega_{p}}{\partial t}\right] \cos 2 \theta
$$

où $A$ et $B$ sont des constantes pour un plasma et une bobine donnés.

La puissance absorbée par le plasma étant indépendante de $\theta$, le signal $\mathrm{S}$ est donc lui aussi proportionnel à $\cos 2 \theta$. Pour faciliter les relevés et en accroître la précision, les mesures de $\mathrm{S}$ ont été effectuées à l'instant de son premier maximum $S_{1}$ qui, nous l'avons vu, a l'amplitude la plus élevée.

On trace alors $S_{1}$ en fonction de $\cos 2 \theta$ et l'on obtient la courbe de la figure 7 : les valeurs de $S_{1}$ pour

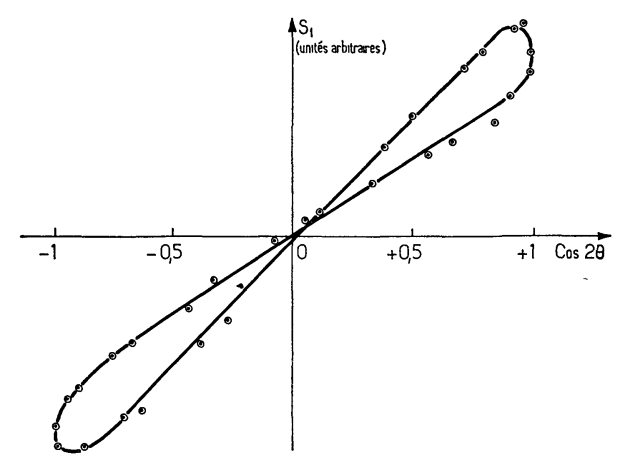

Fig. 7. - Variations de l'amplitude du premier maximum de $\mathrm{S}$ en fonction de $\cos 2 \theta$, où $\theta$ est l'angle mesuré, représenté sur la figure 6.

$\theta$ et $\pi-\theta$ n'ont pas la même valeur bien que correspondant en principe à la même polarisation de l'onde.

Pour rechercher la cause d'une telle dissymétrie, on monte en série sur une transition guide rectangu- 
laire-guide circulaire, le Té de correction, le polariseur, un tronçon de guide circulaire sur lequel est fixée une antenne (fine tige conductrice perpendiculaire à la paroi du guide) et, soit une terminaison adaptée, soit un court-circuit variable. On alimente l'ensemble à bas niveau à $3000 \mathrm{MHz}$ et l'antenne est raccordée à un cristal détecteur branché sur un galvanomètre. Le polariseur étant fixé dans une position $\theta$, on tourne le tronçon de guide circulaire et on note le maximum et le minimum du signal détecté. Le cristal utilisé étant à détection quadratique et l'ellipticité égale au rapport du champ électrique maximum au champ électrique minimum, la racine carrée du rapport du maximum au minimum du signal détecté donne l'ellipticité mesurée $e_{\mathrm{m}}$ de l'onde. L'ellipticité théorique, quant à elle, peut être calculée à partir des coordonnées du vecteur champ électrique selon les axes du polariseur à l'aide de :

$$
\left\{\begin{array}{l}
e_{\mathrm{t}}=\operatorname{tg}\left(\frac{\pi}{4}-\theta\right) \quad \text { pour } \frac{\pi}{2} \leqslant \theta \leqslant \pi \quad \text { (à } k \pi \text { près) } \\
e_{\mathrm{t}}=\operatorname{cotg}\left(\frac{\pi}{4}-\theta\right) \text { pour } \theta \leqslant \theta \leqslant \frac{\pi}{2} \text { (à } k \pi \text { près) } .
\end{array}\right.
$$

On constate alors qu'avec la charge adaptée disposée en bout de guide et pour un angle $\theta$ donné, l'ellipticité $e_{\mathrm{m}}$ est égale à l'ellipticité $e_{\mathrm{t}}$, mais on constate de même qu'en substituant à cette charge un plan de court-circuit, $e_{\mathrm{m}}$ est différente de $e_{\mathrm{t}}$. Ce dernier point n'a d'ailleurs rien pour surprendre compte tenu de l'effet de dépolarisation de l'onde par les réflexions [7], effet qui n'est pas totalement compensé par le Té de correction. Comme on peut le vérifier sur la figure 5 le plasma est lui-même fortement réfléchissant au bout d'un temp négligeable par rapport à celui pour lequel a lieu le premier maximum $S_{1}$ de $\mathrm{S}$ et entraîne donc une dépolarisation au moins partielle de l'onde. La figure 7 représente de ce fait les variations de $\mathrm{S}_{1}$ en fonction de $e_{\mathrm{t}}$ et non en fonction de la váleur réelle $e_{\mathrm{m}}$. Cette dernière grandeur étant évidemment difficile à mesurer pendant le cours de l'expérimentation, le plasma a été simulé par une peinture à l'argent déposée sur la cellule à décharge et $e_{\mathrm{m}}$ mesurée dans ces conditions au banc hyperfréquence. Pour chaque $\theta$ imposé au polariseur on mesure une ellipticité $e_{\mathrm{m}}$ à laquelle on associe à l'aide de (4) l'angle $\theta_{\mathrm{m}}$ qu'aurait le polariseur s'il était parfait (Fig. 8). Ceci permet alors de tracer les variations de $\mathrm{S}_{1}$ en fonction de l'ellipticité réelle, c'est-à-dire en fait en fonction de $\cos 2 \theta_{\mathrm{m}}$. Cette simulation n'est d'ailleurs pas la meilleure possible et il aurait été préférable (mais très difficile à réaliser) de peindre l'intérieur de la cellule; on introduit de cette manière une erreur sur l'angle de phase du coefficient de réflexion ainsi simulé. On s'aperçoit à l'expérience que les meilleurs résultats sont obtenus lorsque l'on déplace vers l'aval la cellule de $5 \mathrm{~mm}$ par rapport à la position qu'elle occupe réellement dans la ligne de propagation. La figure 9 montre alors les variations de $S_{1}$ en

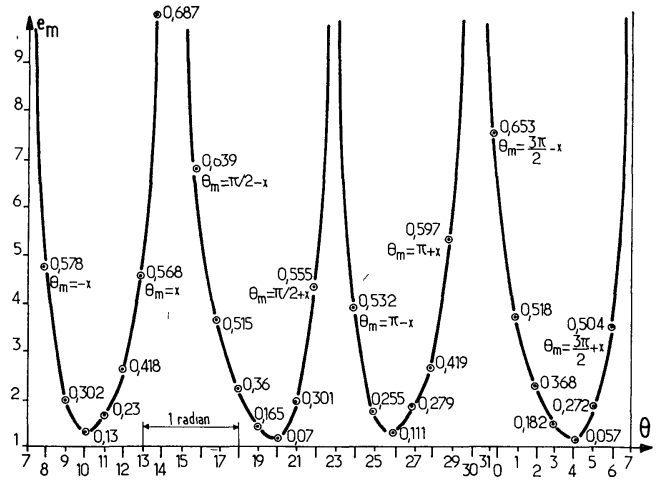

Fig. 8. - Variations en fonction de l'angle $\theta$ de l'ellipticité $e_{\mathrm{m}}$ (mesurée au banc hyperfréquence) lorsque le polariseur est fermé sur la cellule à plasma métallisée extérieurement à la peinture d'argent. Distance entre la cellule et le polariseur : $5 \mathrm{~mm}$.

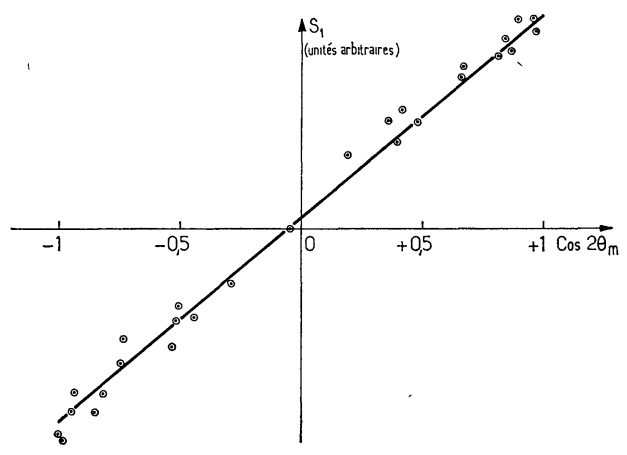

Fig. 9. - Variations de l'amplitude du premier maximum de $\mathrm{S}$ en fonction de $\cos 2 \theta_{\mathrm{m}}$, où $\theta_{\mathrm{m}}$ est l'angle déduit de l'ellipticité mesurée $e_{\mathrm{m}}$ à l'aide de la relation (4).

fonction de $\cos 2 \theta_{\mathrm{m}}$ ainsi obtenues, variations qui respectent bien la loi attendue.

IV. Conclusion. - Nous avons montré que les électrons d'un plasma produisent, sous l'action d'une onde hyperfréquence $(3000 \mathrm{MHz}, \simeq 1 \mathrm{MW})$ polarisée circulairement, une induction magnétique proportionnelle au carré du champ électrique appliqué.

Le signal mesuré aux bornes d'une bobine qui entoure le plasma présente comme prévu deux pics de signes contraires correspondant l'un $\left(S_{1}\right)$ à la montée du signal hyperfréquence, l'autre $\left(S_{2}\right)$ à la descente de ce même signal.

Si on néglige l'effet des parois conductrices sur la croissance de $\mathcal{B}$, pour $\mathrm{S}_{2} \simeq 1 \mathrm{~V}$ et un temps de descente de la $\mathrm{HF} \simeq 0,5 \mu \mathrm{s}$, on trouve que $\mathcal{B} \simeq 5 \times 10^{-3} \mathrm{G}$ et $B \simeq 10^{-5} \mathrm{G} \cdot \mathrm{cm}^{-3}$. L'application pure et simple de la relation (1) au cas $n_{\mathrm{e}} \simeq 10^{12} \mathrm{~cm}^{-3}$ et $E_{0} \simeq 10^{4} \mathrm{~V} / \mathrm{cm}$, donnant $B \simeq 5 \times 10^{-5} \mathrm{G} \cdot \mathrm{cm}^{-3}$; la valeur de l'induction déduite de l'expérience est donc d'un ordre de grandeur comparable à celui obtenu par le calcul.

Remerciements. - Nous remercions Y. Pomeau pour d'utiles discussions concernant ce travail. 


\section{ANNEXE}

Calcul de l'induction $\mathfrak{B}$ créée par une onde de polarisation quelconque. - A la sortie du polariseur le champ électrique de l'onde a pour composantes suivant les axes $\mathrm{O} x$ et $\mathrm{O} y$ (Fig. 6) les valeurs indiquées par (2). Dans le plasma, et à cause des effets de polarisation dus à une densité électronique élevée $\left(\omega_{\mathrm{p}} \simeq \omega\right)$, le champ de l'onde a une valeur $E_{0}$ différente de $E_{\mathrm{i}}$. Sous l'action de ce champ les électrons effectuent des trajectoires elliptiques, somme de deux trajectoires circulaires parcourues en sens contraire et auxquelles on peut associer les vecteurs position $\mathbf{r}_{1}, \mathbf{r}_{2}$ et les vecteurs vitesse $\mathbf{v}_{1}, \mathbf{v}_{2}$ avec $\mathbf{r}=\mathbf{r}_{1}+\mathbf{r}_{2}$ et $\mathbf{v}=\mathbf{v}_{1}+\mathbf{v}_{2}$. Les projections sur $\mathrm{O} x$ et $\mathrm{O} y$ de ces vecteurs sont données par :

$r_{1 x}=\frac{e E_{0}}{m \omega^{2}} \cos \theta \cos \omega t \quad r_{2 x}=-\frac{e E_{0}}{m \omega^{2}} \sin \theta \cos \omega t$

$r_{1 y}=\frac{e E_{0}}{m \omega^{2}} \cos \theta \sin \omega t \quad r_{2 y}=\frac{e E_{0}}{m \omega^{2}} \sin \theta \sin \omega t$

$v_{1 x}=-\frac{e E_{0}}{m \omega} \cos \theta \sin \omega t \quad v_{2 x}=\frac{e E_{0}}{m \omega} \sin \theta \sin \omega t$

$v_{2 x}=\frac{e E_{0}}{m \omega} \cos \theta \cos \omega t \quad v_{2 y}=\frac{e E_{0}}{m \omega} \sin \theta \cos \omega t$.

Le moment magnétique d'un électron étant donné par $\mathbf{m}_{0}=(e / 2)(\mathbf{v} \times \mathbf{r})$, on trouve lorsqu'il est soumis à un tel champ :

$$
\mathbf{m}_{0}=\frac{e}{2 \omega}\left(\frac{e E_{0}}{m}\right)^{2}\left(\cos ^{2} \theta-\sin ^{2} \theta\right) \mathbf{u}
$$

où $\mathbf{u}$ est le vecteur unitaire porté par l'axe $\mathrm{O} z$. L'aimantation par unité de volume ( $n$ électrons) est donc de la forme :

$$
\mathbf{B}=\mu_{0} n \mathbf{m}_{0}=\left(\alpha E_{0}^{2} \cos ^{2} \theta-\alpha E_{0}^{2} \sin ^{2} \theta\right) \mathbf{u} .
$$

Ce résultat peut être obtenu de la manière équivalente suivante : le moment magnétique de l'électron est donné par $m_{0}=S_{0} i$ où $S_{0}$ est la surface de l'ellipse parcourue et $i$ le courant associé au mouvement de l'électron $(i=e \omega / 2 \pi)$. La surface $S_{0}$ de l'ellipse est égale à la différence des surfaces associées à une onde circulaire droite $S_{0 \mathrm{D}}$ et à une onde circulaire gauche $S_{0 \mathrm{G}}$ ayant même fréquence angulaire $\omega$ et respectivement des amplitudes de champ $E_{0} \cos \theta$ et $E_{0} \sin \theta$. Le moment angulaire $m_{0}$ est donc donné par :

$$
m_{0}=S_{0} i=\left(S_{0 \mathrm{D}}-S_{0 \mathrm{G}}\right) i=m_{0 \mathrm{D}}-m_{0 \mathrm{G}}
$$

où $m_{0 \mathrm{D}}$ et $m_{0 \mathrm{G}}$ sont respectivement le moment magnétique associé à l'onde circulaire droite et à l'onde circulaire gauche. Compte tenu de ceci, montrer que $m_{0}$ varie comme $\cos 2 \theta$ montre également la proportionnalité de $\mathcal{B}$ à $E_{0}^{2}$.

L'aimantation totale du plasma est donc de la forme

$$
\mathfrak{B}=\beta E_{0}^{2} \cos ^{2} \theta \mathbf{u}
$$

où $\beta$ dépend de la fréquence de l'onde et de la densité du plasma.

Un tel calcul néglige la vitesse initiale $\mathbf{v}_{0}$ de l'électron mais il est facile de montrer que cette hypothèse ne modifie pas le résultat obtenu. En effet, dans un référentiel $\left(\begin{array}{lll}x^{\prime} & y^{\prime} & z^{\prime}\end{array}\right)$ se déplaçant à la' vitesse $\mathbf{v}_{0}$ par rapport à $(x y z)$, l'électron a la trajectoire elliptique que nous avons calculée et y crée donc le même moment magnétique. Compte tenu de la vitesse des électrons $(v \ll c)$, la valeur de l'induction magnétique $\mathbf{B}$ est très sensiblement la même dans les deux référentiels. Il a par ailleurs été supposé que le champ $E_{0}$ est uniforme dans la direction de propagation et dans un plan transverse. Le gradient longitudinal de $E_{0}$ dépend de l'amortissement de l'onde par le plasma mais il ne modifie pas la forme des variations de $\mathcal{B}$ avec $\theta$ si on admet que la longueur d'atténuation de l'onde ne dépend pas de cet angle ; l'expérience permet assez bien de vérifier ce dernier point. En ce qui concerne la répartition radiale du champ électrique $E_{0}$, elle a la configuration du mode $\mathrm{TE}_{11}$ utilisé et il y a donc lieu de tenir compte de son inhomogénéité.

A la sortie du polariseur, le champ électrique $E_{\mathrm{i}}$ a pour composantes suivant les axes $\mathrm{O} x$ et $\mathrm{O} y$ déjà utilisés les valeurs suivantes :

$$
\begin{gathered}
E_{x}=E_{i} \sqrt{2}\left[\cos \varphi \frac{J_{1}(\rho)}{\rho} \cos \left(\varphi+\theta+\frac{\pi}{4}\right)+\right. \\
\left.+\sin \varphi J_{1}^{\prime}(\rho) \sin \left(\varphi+\theta+\frac{\pi}{4}\right)\right] \cos \omega t \\
E_{v}=E_{i} \sqrt{2}\left[\cos \varphi \frac{J_{1}(\rho)}{\rho} \sin \left(\varphi+\theta+\frac{\pi}{4}\right)-\right. \\
\left.-\sin \varphi J_{1}^{\prime}(\rho) \cos \left(\varphi+\theta+\frac{\pi}{4}\right)\right] \sin \omega
\end{gathered}
$$

où $\varphi$ est l'angle du vecteur position $r$ du point considéré avec la direction de polarisation, $J_{1}(\rho)$ la fonction de Bessel d'ordre 1 et $\rho=2 \pi \mathrm{r} / \lambda_{\mathrm{c}}$ avec $\lambda_{\mathrm{c}}=3,41 \mathrm{a}$, a étant le rayon du guide.

La détermination du moment magnétique créé dans cette configuration de champ suppose,' comme précédemment, le calcul des vecteurs position et vitesse des électrons et donc la résolution d'équations différentielles du second ordre dont les solutions ne peuvent maintenant être obtenues sans difficultés.

Un tel calcul peut en fait être évité à condition de remarquer que les fonctions $J_{1}(\rho) / \rho$ et $J_{1}^{\prime}(\rho)$ ont des valeurs comparables au voisinage de l'axe et que les « rayons de giration » des électrons dans les conditions de l'expérience sont petits devant le rayon du guide.

Jusqu'à des valeurs de $\rho$ de l'ordre de 0,6 , les composantes suivant ox et oy peuvent en effet s'écrire : $E_{x}=\delta E_{i}(\cos \theta-\sin \theta) \cos \omega t$ et $E_{y}=\delta E_{i}(\cos \theta+\sin \theta) \sin \omega t$ avec $\delta \simeq 0,5$. Un tel champ impose aux électrons une trajectoire elliptique 
qui crée une induction magnétique dont les variations en $\theta$ sont, ainsi qu'il a été calculé précédemment, de la forme $\cos 2 \theta$.

En outre, pour $\rho \gtrsim 0,6$ le calcul se simplifie également car, pendant une période de l'onde $(2 \pi / \omega)$, les électrons décrivent une trajectoire dont l'extension est petite devant celle du guide (dans un champ homogène le rayon de giration est de l'ordre de $0,5 \mathrm{~mm}$ pour un champ de $10^{6} \mathrm{~V} / \mathrm{m}$ ) : les fonctions en $\varphi$ et en $\rho$ dans l'expression de $\mathbf{E}_{i}$ peuvent ainsi être considérées comme stationnaires. On calcule alors le vec- teur position $\mathbf{r}$ et le vecteur vitesse $\mathbf{v}$ d'un électron et l'on obtient :

$$
\begin{array}{r}
\mathbf{B}=\left[\mu_{0} \frac{e^{3}}{2 m^{2} \omega^{3}} \cdot \frac{\pi}{4}\left(\frac{\lambda_{\mathrm{c}}}{2 \pi}\right)^{2} \cdot \int_{0}^{1,84} n(\rho) J_{2}^{2}(\rho) \rho \mathrm{d} \rho\right] \times \\
\times E_{0}^{2} \cos 2 \theta
\end{array}
$$

où $n(\rho)$ représente les variations radiales de la densité électronique et $J_{2}(\rho)$ la fonction de Bessel d'ordre 2 . On retrouve bien comme précédemment une loi en $E_{0}^{2} \cos 2 \theta$.

\section{Bibliographie}

[1] Pershan (P. S.), Phys. Rev., 1963, 130, 919.

[2] Pershan (P. S.), Vanderziel (J. P.), Malmstrom (L. P.), Phys. Rev., 1966, 143, 574.

[3] Vanderziel (J. P.), Pershan (P. S.), Malmstrom (L. D.), Phys. Rev. Letters, 1965, 15, 190.

[4] Pomeau (Y.), Quemada (D.), C. R. Acad. Sci., Paris, 1967, Sér. B 264, 517.
[5] Deschamps (J.), Fitaire (M.), Lagoutte (M.), Phys. Rev. Letters, 1970, 25, 1330.

[6] Bottiglioni (F.), Plasma Phys., 1970, 12, 131.

[7] Deschamps (J.), Fitaire (M.), Pagnon (D.), Rev. Phys. Appl., 1970, 5, 283. 